\title{
Endemic Crimean Congo Hemorrhagic Fever in Pakistan
}

"In times of stress and danger such as come about as the result of an epidemic, many tragic and cruel phases of human nature are brought out, as well as many brave and unselfish ones."

William Crawford Gorgas

Crimean Congo hemorrhagic fever is endemic in certain parts of world. It is a zoonotic disease and reservoirs are domestic and wild animals. It spreads by vector Hyalomma tick or contact with infected animals or people or infected secretions. The clinical disease spectrum includes fever with flu like symptoms, hemo-rrhages and mortality rate of $10-40 \%$. The incubation period is $1-3$ days after a tick bite or $5-6$ days fol-lowing exposure to infected blood or tissues. The $f l u$ - like symptoms may resolve after one week. In up to $75 \%$ of cases, however, signs of hemorrhage appear within 3-5 days of the onset of illness in the form of skin bruises, nose bleeds, vomiting, and black stools. The liver becomes swollen and tender. Patients usually begin to show signs of recovery after $9-10$ days from when symptoms presented. ${ }^{1} 10-40 \%$ of the cases result in mortality by the end of the second week of illness and may be attributed by hemorrhagic shock, hypovolemia, septacaemia, acute kidney failure, and acute respiratory distress syndrome. ${ }^{2}$

Pakistan has witnessed severe outbreaks in 2009 and 2010. In 2009, epidemic of Crimean Congo hemorrhagic fever was reported from Baluchistan. In September 2010, an outbreak was reported in Pakistan's Khyber Pakhtunkhwa province. Poor diagnosis and record keeping has caused the extent of the epidemic to be uncertain, though some reports indicate over 100 cases, with a case - fatality rate above $10 \%$. Crimean - Congo haemorrhagic fever is declared endemic in Pakistan. Human infections caused by the CrimeanCongo haemorrhagic virus have been occurring throughout the year and in wide geographic areas of the country. The seasonal spike has been reported this year and guidelines for prevention in public and health care providers are formulated. However, clear and rational policies from law enforcement agencies to avoid spread from endemic foci to other non-endemic areas through transportation of animals or contact with infected cases especially during Eid festivals are still needed. The transportation of animals is greatly increased during Eid festival in Pakistan and risk of epidemic is also increased. Law enforcement and Agricultural regulations require de-ticking farm animals before transportation or delivery for slaughter. Protocols for safety during slaughter and disposal of infected wastes should be formulated and implemented. In the case when feverish patients with evidence of bleeding are reported, emergency preparations for resuscitation or intensive care are required urgently. Moreover guidelines regarding suspected cases quarantine, body secretions and wastes isolation and disposal in health care facilities and standard precautions for laboratory workers, nursing staff and doctors should be adopted.

Surveillance and laboratory diagnosis for early detection of cases, infection control measures in health care facilities and risk communication should be stre-ngthened especially in high risk areas in the country. Sero-prevalence of antibodies against Crimean Congo hemorrhagic virus in our community is still unknown.

Preventive steps are simple but awareness in masses about Crimean congo hemorrhagic fever is the most important step. Some of the important steps for prevention are:

- Use a repellent containing 20\% - 30\% DEET or $20 \%$ Picaridin. Re-apply according to manufacturer's directions.

- Wear neutral - coloured and light - weight clothes, long - sleeved shirts and full pants. Tuck pants into socks for further protection.

- Apply a permethrin spray or solution to clothing and gear.

- When walking through grass lands avoid tall grasses and shrubs. 
- Carefully examine body, clothing, gear, and animals for ticks.

- Apply sunscreen first followed by the repellent and preferably 20 minutes later.

- Avoid coming into contact with the blood or tissues of animals. Healthcare practitioners should take appropriate infection control measures to prevent infection. Standard operating procedures to handle infectious materials and suspected cases should be displayed in clinical settings.

- Laboratory staff should wear protective gear and waste disposal should be according to the protocols.

There is no effective commercially available vaccine or chemoprophylaxis against Crimean-Congo Hemorrhagic Fever. Thus efforts should be directed to prevent this disease by awareness in masses. Moreover, sero-prevalence in general public as well as in specific groups including health care providers, labora-tory workers, butchers, veterans and surgeons should be detected by screening and later on confirmed by Enzyme Linked Immunosorbant Assay (ELISA). Early case detection, quarantine of susceptible cases and adoption of standard protocols during management of patients can decrease the spread of this deadly virus.

\section{References}

1. Bente DA, Forester NL, Watts DM, McAuley AJ, Whitehouse CA, Bray M. Crimean - Congo hemorrhagic fever: history, epidemiology, pathogenesis, clinical syndrome and genetic diversity. Antiviral Res. 2013; 1663542 (13): 00193-9.doi:10.1016/j.antiviral.2013.07.006

2. Carroll SA, Bird BH, Rollin PE, Nichol ST. Ancient common ancestry of Crimean - Congo hemorrhagic fever virus. Mol Phylogenet Evol. 2010; 55 (3): 11031110. doi:10.1016/j.ympev.2010.01.006 


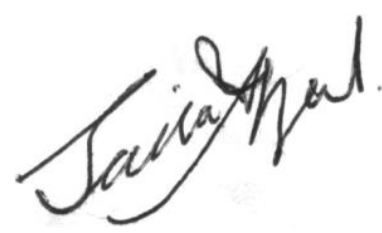

Dr. Saira Afzal

Editor Annals of KEMU 